\title{
A Novel Anatomic Titanium Mesh Cage for Reducing the Subsidence Rate After Anterior Cervical Corpectomy: A Finite Element Study
}

\section{Yuhang Wang}

Honghui Hospital

Qinpeng Zhao

Honghui Hospital

Yongyuan Zhang

Honghui Hospital

Haiping Zhang

Honghui Hospital

Baorong He

Honghui Hospital

Liang Yan

Honghui Hospital

Dingjun Hao

Honghui Hospital

Biao Wang ( $\square$ wangbiaowb1987@126.com )

Department of Spine Surgery, Xi'an Jiaotong University College of Medicine, Honghui Hospital, No. 76 Nanguo Road, Xi'an 710054, Shaanxi, China https://orcid.org/0000-0001-6631-9127

\section{Research article}

Keywords: Titanium mesh cage, Cervical spine, ACCF, Subsidence rate

Posted Date: August 20th, 2020

DOI: https://doi.org/10.21203/rs.3.rs-53986/v1

License: (c) (i) This work is licensed under a Creative Commons Attribution 4.0 International License.

Read Full License

Version of Record: A version of this preprint was published at Scientific Reports on July 28th, 2021. See the published version at https://doi.org/10.1038/s41598-021-94787-0. 


\section{Abstract}

Background: Fusion with a titanium mesh cage (TMC) has become popular as a conventional method after cervical anterior corpectomy, but postoperative TMC subsidence has often been reported in the literature. We designed a novel anatomic cervical TMC to reduce the postoperative subsidence rate. This finite element study aims to investigate the anti-subsidence performance of a novel anatomic TMC and provide a theoretical basis for clinical application.

Methods: According to the test process specified in the ASTM F2267 standard, three-dimensional finite element analysis was used to compare the anti-subsidence characteristics of a traditional TMC (TTMC) and novel TMC (NTMC). Abaqus software was used for the mesh, and the modulus of elasticity, Poisson's ratio and other material coefficients of each part were input into the model. Solidworks software was used to establish the TTMC and NTMC models and construct the two three-dimensional finite element models of TMC subsidence testing mentioned above. Through analysis, the relationships between the stiffness of the intervertebral body fusion device $(\mathrm{Kd})$ and the stiffness of the polyurethane foam blocks (Ks) of the TTMC and NTMC were derived, respectively.

Results: After fitting the linear segment, the Kd values of the TTMC and NTMC were $37314 \mathrm{~N} / \mathrm{mm}$ and $89124 \mathrm{~N} / \mathrm{mm}$, respectively. After calculation, the Ks values of the TTMC and NTMC were $653.83 \mathrm{~N} / \mathrm{mm}$ and $995.95 \mathrm{~N} / \mathrm{mm}$, respectively. The Kp values of the TTMC and NTMC were calculated to be 665.5 $\mathrm{N} / \mathrm{mm}$ and $1007.2 \mathrm{~N} / \mathrm{mm}$ according to the formula.

Conclusion: The reported novel anatomic titanium mesh cage (NTMC) significantly improved the antisubsidence performance after ACCF, which was approximately $51.3 \%$ higher than that of the traditional titanium mesh cage.

\section{Introduction}

Anterior cervical corpectomy and fusion (ACCF) is a common and effective treatment modality for various cervical disorders, including cervical spondylosis myelopathy, ossified posterior longitudinal ligaments, trauma, tumors, deformity corrections, infections and rheumatoid arthritis, especially when the disease involves one or two vertebral levels [1-4]. ACCF has been widely used as a well-tolerated approach with satisfactory postoperative outcomes, owing to the direct decompression of the spinal cord and nerve root and immediate stabilization of the affected segments $[5,6]$. However, reconstruction after corpectomy is challenging and may be accomplished with implantation of autografts, allografts or bone substitutes $[7,8]$.

The use of a titanium mesh cage (TMC) with local bone grafting has become the main method for cervical reconstruction during ACCF surgery [9]. Although this method avoids the complications of the bone donor site, maintains immediate anterior column stability with good biocompatibility, and has a high fusion rate, the incidence of postoperative TMC subsidence reported in the literature is as high as $28.6 \%-93.3 \%[6,10-12]$. TMC subsidence may be correlated with poor clinical efficacy or poor 
neurological recovery. Severe subsidence will lead to symptom recurrence, deterioration of nerve function, failure of internal fixation and kyphosis of the cervical spine $[13,14]$. There are many risk factors related to TMC subsidence, among which patients' own conditions and operation reasons can be avoided by case screening and operation technology improvements. However, the inherent defects of TMC itself are urgent problems that need to be solved in clinical practice [12].

At present, the contact area between the TMC and vertebral end plate is limited. The upper and lower end plates of cervical vertebrae are all irregularly curved. After TMC implantation, they are inlaid with the end plate by the dentate edge, and the adhesiveness is poor. The contact area between the TMC and end plate is small, which is point-to-face contact. Moreover, this kind of point contact causes uneven stress distribution and relatively concentrated stress, which easily causes TMC subsidence after surgery. Therefore, it is of great significance to find a new type of TMC that is in line with the anatomical structure of the cervical vertebrae of the patients, to change the point-to-face contact into the face-to-face contact, and to avoid postoperative TMC subsidence and related complications.

For this reason, an anatomic TMC system was developed, and its mechanical properties were analyzed using a three-dimensional finite element method to determine whether this novel TMC could effectively reduce the risk of subsidence after ACCF surgery and provide a reference for further biomechanical experiments.

\section{Materials And Methods}

\section{Design of the novel TMC}

The new type of anatomical TMC for the cervical spine adopts a circular hollow column structure, which is consistent with human engineering mechanics (Fig. 1). The two ends of the TMC were provided with the upper edge curved surface structure and the lower edge curved surface structure integrated with the TMC main body. In addition, the TMC main body has a hollow cylindrical structure penetrating the upper and lower curved surface structures, and a plurality of visible holes connecting the hollow cylindrical structure was arranged on the main body surface. Based on the analysis of a large number of CT scan data of cervical vertebrae, the size of the novel TMC was classified in a step-by-step mode, and the curvature of the upper and lower edge curved surface structures matched with the anatomical data of the end plates. The upper edge curved surface structure was matched with the lower end plate of the fusion upper cervical vertebra to form face-to-face contact; the lower edge curved surface structure was matched with the upper end plate of the fusion lower cervical vertebra to form face-to-face contact.

\section{Subsidence testing standard}

In this study, three-dimensional finite element analysis was used to compare the anti-subsidence characteristics of the traditional TMC (TTMC) and novel TMC (NTMC). The two TMC models are shown in Fig. 2. The TMC, as an intervertebral body fusion device, and the subsidence testing, as described in 
ASTM F2267(2011), were intended to characterize the propensity of a TCM to subside into the vertebral body end plates [15]. By modeling the subsidence testing systems as two springs in series, one can derive the relationship between the stiffness of the intervertebral body fusion device and the stiffness of the polyurethane foam blocks (simulated vertebral bodies). The relative propensity of a device to subside is quantified by a stiffness measurement, $\mathrm{Kp}(\mathrm{N} / \mathrm{mm})$, as follows: $\mathrm{Kp}=\left(\mathrm{Ks}^{\star} \mathrm{Kd}\right) /(\mathrm{Kd}-\mathrm{Ks}) . \mathrm{Kd}$ is the stiffness of the intervertebral body fusion device, and $\mathrm{Ks}$ is the stiffness of the system.

\section{Research model establishment and loading}

The height of both TMCs was set as $25 \mathrm{~mm}$, the TTMC (Medtronic Sofamor Danek, Memphis, TN) thickness was set as $1.3 \mathrm{~mm}$, and the outer diameter was set as $13 \mathrm{~mm}$. The thickness of the middle part of the NTMC was $1.3 \mathrm{~mm}$, and the outer diameter was $13 \mathrm{~mm}$. In terms of size, the two TMCs were very close. The NTMC adopted a full fit design at the interface with bone tissue.

According to the test process specified in the ASTM F2267 standard, first, the rigid pressure clamp was selected to load the displacement load along the vertical direction, the size was $5 \mathrm{~mm}$, and the measured rigidity of this process was recorded as $\mathrm{Kd}$. Then, the rigid pressure clamp was changed to grade 15 polyurethane foam blocks (intended to replicate the compression properties of trabecular bone). The loading process described above was repeated, and the measured stiffness of this process was recorded as Ks. Kp was calculated according to the above formula. A higher Kp measurement is generally expected to indicate that the device is more resistant to subsidence into a vertebral body.

Taking the NTMC as an example, the boundary conditions and load settings were illustrated. The size of the upper and lower grade 15 polyurethane foam blocks was $40 * 40 * 60(\mathrm{~mm})$, and the upper and lower surfaces of the blocks were selected to establish coupling constraints, as shown in Fig. 3. RP1 was coupled with the upper surface of the NTMC, and RP2 was coupled with the lower surface of the NTMC. The downward displacement load of $5 \mathrm{~mm}$ was loaded on RP1, and the other degrees of freedom were constrained. All degrees of freedom on RP2 were constrained. The upper and lower polyurethane foam blocks and NTMC were set as face-to-face contact, the contact property was vertical hard contact, and the horizontal direction was set as "rough" to ensure that no sliding occurred in the horizontal direction during the loading process. Abaqus software was used for the mesh, and the modulus of elasticity, Poisson's ratio and other material coefficients of each part were input into the model (Table 1). Solidworks software was used to establish the TTMC and NTMC models and construct the two threedimensional finite element models of TMC subsidence testing described above. 
Table 1

Material properties of the components of FE model

\begin{tabular}{|llll|}
\hline Description & Young's Modulus (MPa) & Poisson's Ratio & Yield strength (MPa) \\
\hline TTMC & 110,000 & 0.3 & 830 \\
\hline NTMC & 110,000 & 0.3 & 830 \\
\hline grade 15 blocks & 123 & 0.3 & 4.9 \\
\hline
\end{tabular}

\section{Results}

\section{TTMC analysis results}

The stress distribution and the force displacement curve of Kd are shown in Fig. 4. Although the loading was $5 \mathrm{~mm}$, because the upper and lower pressure blocks were rigid bodies, the calculation stopped due to element distortion when the overall compression displacement was $0.2873 \mathrm{~mm}$. However, at this time, the stiffness $\mathrm{Kd}$ could be obtained, and the whole TTMC yielded. After fitting the linear segment, the Kd value was $37314 \mathrm{~N} / \mathrm{mm}$. The stress distribution and the force displacement curve of Ks are shown in Fig. 5. When the total displacement was loaded to $1.232 \mathrm{~mm}$, the TTMC sank into the polyurethane foam blocks, and the Ks calculation stopped. The subsidence and displacement nephogram of the TTMC is shown in Fig. 6. At this time, the Ks value obtained was $653.83 \mathrm{~N} / \mathrm{mm}$.

The Kp value of the TTMC was calculated to be $665.5 \mathrm{~N} / \mathrm{mm}$ according to the formula.

\section{NTMC analysis results}

The stress distribution and the force displacement curve of Kd are shown in Fig. 7. Similar to the TTMC, although the loading was $5 \mathrm{~mm}$, because the upper and lower pressure blocks were rigid bodies, the calculation stopped due to element distortion when the overall compression displacement was $0.317 \mathrm{~mm}$. However, at this time, the stiffness Kd could be obtained, and the whole NTMC yielded. After fitting the linear segment, the KD value was $89124 \mathrm{~N} / \mathrm{mm}$. The stress distribution and the force displacement curve of Ks are shown in Fig. 8. When the total displacement was loaded to $0.75 \mathrm{~mm}$, the NTMC sank into the polyurethane foam blocks, and the Ks calculation stopped. The subsidence and displacement nephogram of the NTMC is shown in Fig. 9. At this time, the Ks value obtained was $995.95 \mathrm{~N} / \mathrm{mm}$.

The Kp value of the NTMC was calculated to be $1007.2 \mathrm{~N} / \mathrm{mm}$ according to the formula.

\section{Improvement rate of anti-subsidence performance}

The calculation formula of the anti-subsidence performance improvement rate is $\left(\mathrm{Kp}_{(\mathrm{NTMC})^{-}}\right.$ $\left.\mathrm{Kp}_{(\mathrm{TTMC})}\right) / \mathrm{Kp}_{\text {(TTMC) }}$. After calculation, the anti-subsidence performance of the NTMC was approximately $51.3 \%$ higher than that of the TTMC. 


\section{Discussion}

ACCF has long been a classic operation in anterior cervical surgery, and its curative effect is stable and reliable, but the structural integrity of the cervical spine after corpectomy remains a challenge $[1,8,16]$. The use of an autologous bone graft from the iliac crest is the ideal solution for the reconstruction of corpectomy defects. However, donor site morbidity has been reported in up to $25 \%$ of patients undergoing this procedure [17], and in the cervical spine, postoperative complications such as pseudoarthrosis, graft displacement, fracture and deformity also occur occasionally [18]. Allografts and bone substitutes can avoid the morbidity associated with graft harvesting, but their use has been questioned due to the delayed union and low fusion rates $[6,19,20]$. Currently, different intervertebral body fusion devices have been developed to maximize anterior column stability, avoid donor-site morbidity, improve biocompatibility, and reduce instrumentation-related morbidity [6].

Among them, the TMC has been highly praised by most surgeons with a high fusion rate [21]. Thalgott et al [22] reported a $100 \%$ fusion rate for multiple-level cervical corpectomy fusion using the TMC with a local bone graft. However, the TMC subsidence rate is very high and may cause instability, reconstruction failure and neurological deterioration. In a large sample size study by Chen et al [23], TMC subsidence was observed in 239 of 300 patients (79.7\%) after ACCF surgery. In another over 8-year follow-up study by Hu et al [24], among the 52 patients with single-level anterior corpectomy, the probability of TMC subsidence of $>3 \mathrm{~mm}$ was as high as $40.4 \%$. Through the analysis of the traditional TMC, it is clear that the main reason for subsidence is that the shape of the upper and lower ends of the TMC is not consistent with that of the end plate, and because the end surface of the TMC needs to be cut during the operation and the contact points are often sharp, it is easy to produce stress concentration and puncture the bony end plate. A TMC with sharp edges increases primary stability and resistance to early anterior displacement by cutting through the end plate, but it may also increase the chance of late subsidence [25]. This kind of point surface contact easily causes TMC subsidence.

In this study, we invented a novel type of anatomical TMC for the cervical spine. The new curved structure of the upper and lower edges of the NTMC can perfectly fit with the upper and lower end plates of the cervical spine, and it can truly achieve ideal face-to-face contact. Theoretically, the upper and lower contact surfaces designed by the NTMC according to the surface characteristics of the cervical endplate increase the contact area between the NTMC and upper and lower vertebrae. When the cervical vertebrae move, the NTMC can distribute the load onto the surface of the upper and lower vertebrae better and improve the anti-subsidence performance of the TMC.

The finite element method is an ideal tool to study the biomechanics of the spine and implanted medical devices [26, 27]. This study is based on the subsidence testing standard of the intervertebral body fusion device, ASTM F2267, to analyze the anti-subsidence characteristics of the NTMC using the finite element method. After calculation, the KP of the TTMC was $665.5 \mathrm{~N} / \mathrm{mm}$, while the KP of the NTMC with face-toface contact to the end plates was increased to $1007.2 \mathrm{~N} / \mathrm{mm}$. The Kp value is the benchmark to measure the subsidence tendency of the TMC. The larger the KP value is, the smaller the tendency of the 
TMC to sink into the vertebral body. In contrast, the smaller the KP value is, the greater the tendency of the TMC to sink into the vertebral body. Our results show that the NTMC does not easily sink compared with the TTMC, and the anti-subsidence performance of the NTMC is significantly improved by $51.3 \%$.

Owing to the sharp footprints of the TTMC and in order to improve the contact relationship between the vertebral body and TMC, the use of end-caps has been recently suggested to increase the contact area and reduce the subsidence rate although, in theory, the TMC with end-caps also achieves face-to-face contact. However, it is essentially different from the NTMC described by us. Because the end plate is a curved structure and the TMC with end caps is a planar structure, the end cap cannot form good adhesion with the end plate. Chen et al [23] suggested that end caps did not sufficiently increase the contact area to resist subsidence due to the different orientations of the TMC surface and the endplates; conversely, bone fusion was delayed because of the loss of bony contact. Hur et al [28] conducted a comparative study on 84 patients with single-segment ACCF in the six-year period. Subsidence was less frequent in the TMC with end-cap group (34.2\%) than in the TMC without end-cap group (52.1\%). However, its anti-subsidence performance was only improved by $34.4 \%$, which is significantly less than the improvement rate of the NTMC by $51.3 \%$. The reason may be that the curved structure of the upper and lower edges of the NTMC can effectively fit with the upper and lower end plates to provide better antisubsidence performance.

This study has the following shortcomings as a finite element study of the anti-subsidence performance of the NTMC. First, due to the requirements of finite element technology, in the process of modeling, bone tissue and the end face of the TMC must be $100 \%$ integrated, while the TTMC has difficultly achieving such an ideal state in the actual operation process, so the actual results of the TTMC may be worse than the simulation results. Second, to facilitate the popularization and application of the NTMC, the size of the NTMC is divided into different types in a stepped mode based on the analysis of CT scan data of cervical vertebrae. In theory, the individualized 3D-printed NTMC based on the patient's cervical CT data will have better matching and anti-subsidence performance. Of course, according to the existing structure of the NTMC, a 3D printing model can also be made. In addition, in this study, only the anti-subsidence characteristics of the NTMC were defined, but its stability and effectiveness still need to be further verified.

\section{Conclusion}

According to the predicted results of this finite element analysis, the reported novel anatomic titanium mesh cage significantly improved the anti-subsidence performance after ACCF, which was approximately $51.3 \%$ higher than the performance of the traditional titanium mesh cage.

\section{Abbreviations}

TMC: Titanium mesh cage; TTMC: Traditional titanium mesh cage; NTMC: Novel titanium mesh cage; Kd: Stiffness of the intervertebral body fusion device; Ks: Stiffness of the polyurethane foam blocks; Kp: = 
(Ks* Kd)/(Kd-Ks); ACCF: Anterior cervical corpectomyand fusion; CT: Computed tomography; ASTM: American Society of Testing Materials; RP: polyurethane foam block.

\section{Declarations}

\section{Acknowledgements}

We are thankful for the support of the Department of Spine Surgery, Xi'an Jiaotong University College of Medicine, Honghui Hospital.

\section{Authors' contributions}

YW, BW and LK carried out the finite element analysis and drafted the manuscript. BW, HG, LY and DH participated in the study design and discussion of the clinical results. BW, YW and $\mathrm{HZ}$ constructed the finite element models, performed the biomechanical analysis. All of the authors read and approved the final manuscript.

\section{Funding}

This work was supported by the National Natural Science Foundation of China (No.81802167),Natural Science Research Plan in Shaanxi Province of China (No.2019JM197), and Sanitation and Health Research Fund of Shaanxi Province (2018E001).Fundings include the cost of collecting the fellow-up data and publication fee.

\section{Availability of data and materials}

All relevant data was presented within the manuscript and the datasets usedand/or analyzed during the current study are available from thecorresponding author on reasonable request.

\section{Ethics approval and consent to participate}

Not applicable.

\section{Consent for publication}

Not applicable.

\section{Competing interests}

The authors have no competing interests.

\section{Author details}

Department of Spine Surgery, Xi'an Jiaotong University College of Medicine, Honghui Hospital, No. 76 Nanguo Road, Xi'an 710054, Shaanxi, China 


\section{References}

1. Zhang $\mathrm{Y}$, Liu H, Yang H, Pi B. Anterior cervical corpectomy and fusion versus discectomy and fusion for the treatment of two-level cervical spondylotic myelopathy: analysis of sagittal balance and axial symptoms. Int Orthop. 2018;42(8):1877-82.

2. Gao W, Wang B, Hao D, et al. Surgical Treatment of Lower Cervical Fracture-Dislocation with Spinal Cord Injuries by Anterior Approach: 5- to 15-Year Follow-Up. World Neurosurg. 2018;115:e137-45.

3. Jang JW, Lee JK, Lee JH, Hur H, Kim TW, Kim SH. Effect of posterior subsidence on cervical alignment after anterior cervical corpectomy and reconstruction using titanium mesh cages in degenerative cervical disease. J Clin Neurosci. 2014;21(10):1779-85.

4. Qin R, Chen X, Zhou P, Li M, Hao J, Zhang F. Anterior cervical corpectomy and fusion versus posterior laminoplasty for the treatment of oppressive myelopathy owing to cervical ossification of posterior longitudinal ligament: a meta-analysis. Eur Spine J. 2018;27(6):1375-87.

5. Medow JE, Trost G, Sandin J. Surgical management of cervical myelopathy: indications and techniques for surgical corpectomy. Spine J. 2006;6(6 Suppl):233S-241S.

6. Weber MH, Fortin M, Shen J, et al. Graft Subsidence and Revision Rates Following Anterior Cervical Corpectomy: A Clinical Study Comparing Different Interbody Cages. Clin Spine Surg. 2017;30(9):E1239-45.

7. Zhang Y, Deng X, Jiang D, et al. Long-term results of anterior cervical corpectomy and fusion with nano-hydroxyapatite/polyamide 66 strut for cervical spondylotic myelopathy. Sci Rep. 2016;6:26751.

8. Thalgott JS, Xiongsheng C, Giuffre JM. Single stage anterior cervical reconstruction with titanium mesh cages, local bone graft, and anterior plating. Spine J. 2003;3(4):294-300.

9. Narotam PK, Pauley SM, McGinn GJ. Titanium mesh cages for cervical spine stabilization after corpectomy: a clinical and radiological study. J Neurosurg. 2003;99(2 Suppl):172-80.

10. Fengbin $Y$, Jinhao M, Xinyuan L, Xinwei W, Yu C, Deyu C. Evaluation of a new type of titanium mesh cage versus the traditional titanium mesh cage for single-level, anterior cervical corpectomy and fusion. Eur Spine J. 2013;22(12):2891-6.

11. Lu T, Liang H, Liu C, et al. Effects of Titanium Mesh Cage End Structures on the Compressive Load at the Endplate Interface: A Cadaveric Biomechanical Study. Med Sci Monit. 2017;23:2863-70.

12. Wu J, Luo D, Ye X, Luo X, Yan L, Qian H. Anatomy-related risk factors for the subsidence of titanium mesh cage in cervical reconstruction after one-level corpectomy. Int J Clin Exp Med. 2015;8(5):740511.

13. Daubs MD. Early failures following cervical corpectomy reconstruction with titanium mesh cages and anterior plating. Spine (Phila Pa 1976). 2005;30(12):1402-6.

14. Ji C, Yu S, Yan N, et al. Risk factors for subsidence of titanium mesh cage following single-level anterior cervical corpectomy and fusion. BMC Musculoskelet Disord. 2020;21(1):32.

15. Peck JH, Sing DC, Nagaraja S, Peck DG, Lotz JC, Dmitriev AE. Mechanical performance of cervical intervertebral body fusion devices: A systematic analysis of data submitted to the Food and Drug 
Administration. J Biomech. 2017;54:26-32.

16. Guan L, Hai Y, Yang JC, Zhou LJ, Chen XL. Anterior cervical discectomy and fusion may be more effective than anterior cervical corpectomy and fusion for the treatment of cervical spondylotic myelopathy. BMC Musculoskelet Disord. 2015;16:29.

17. Sawin PD, Traynelis VC, Menezes AH. A comparative analysis of fusion rates and donor-site morbidity for autogeneic rib and iliac crest bone grafts in posterior cervical fusions. J Neurosurg. 1998;88(2):255-65.

18. Wright IP, Eisenstein SM. Anterior cervical discectomy and fusion without instrumentation. Spine (Phila Pa 1976). 2007;32(7):772-5.

19. Floyd T, Ohnmeiss D. A meta-analysis of autograft versus allograft in anterior cervical fusion. Eur Spine J. 2000;9(5):398-403.

20. Malloy KM, Hilibrand AS. Autograft versus allograft in degenerative cervical disease. Clin Orthop Relat Res. 2002;(394):27-38.

21. Wen Z, Lu T, Wang Y, Liang H, Gao Z, He X. Anterior Cervical Corpectomy and Fusion and Anterior Cervical Discectomy and Fusion Using Titanium Mesh Cages for Treatment of Degenerative Cervical Pathologies: A Literature Review. Med Sci Monit. 2018;24:6398-404.

22. Thalgott JS, Xiongsheng C, Giuffre JM. Single stage anterior cervical reconstruction with titanium mesh cages, local bone graft, and anterior plating. Spine J. 2003;3(4):294-300.

23. Chen Y, Chen D, Guo Y, et al. Subsidence of titanium mesh cage: a study based on 300 cases. J Spinal Disord Tech. 2008;21(7):489-92.

24. Hu B, Wang L, Song Y, et al. A comparison of long-term outcomes of nanohydroxyapatite/polyamide66 cage and titanium mesh cage in anterior cervical corpectomy and fusion: A clinical follow-up study of least 8 years. Clin Neurol Neurosurg. 2019;176:25-9.

25. Lee SH, Sung JK. Anterior cervical stabilization using a semi-constrained cervical plate and titanium mesh cage for single level corpectomy. J Clin Neurosci. 2008;15(11):1227-34.

26. Zhang $\mathrm{H}$, Hao D, Sun $\mathrm{H}$, et al. Biomechanical effects of direction-changeable cage positions on lumbar spine: a finite element study. Am J Transl Res. 2020;12(2):389-96.

27. Su X, Shen H, Shi W, Yang H, Lv F, Lin J. Dynamic characteristics of osteoporotic lumbar spine under vertical vibration after cement augmentation. Am J Transl Res. 2017;9(9):4036-45.

28. Hur JW, Ryu KS, Ahn S, Kim JS, Chung HJ, Song MS. Comparative Analysis of 2 Different Types of Titanium Mesh Cage for Single-level Anterior Cervical Corpectomy and Fusion in Terms of Postoperative Subsidence and Sagittal Alignment. Clin Spine Surg. 2020;33(1):E8-13.

\section{Figures}




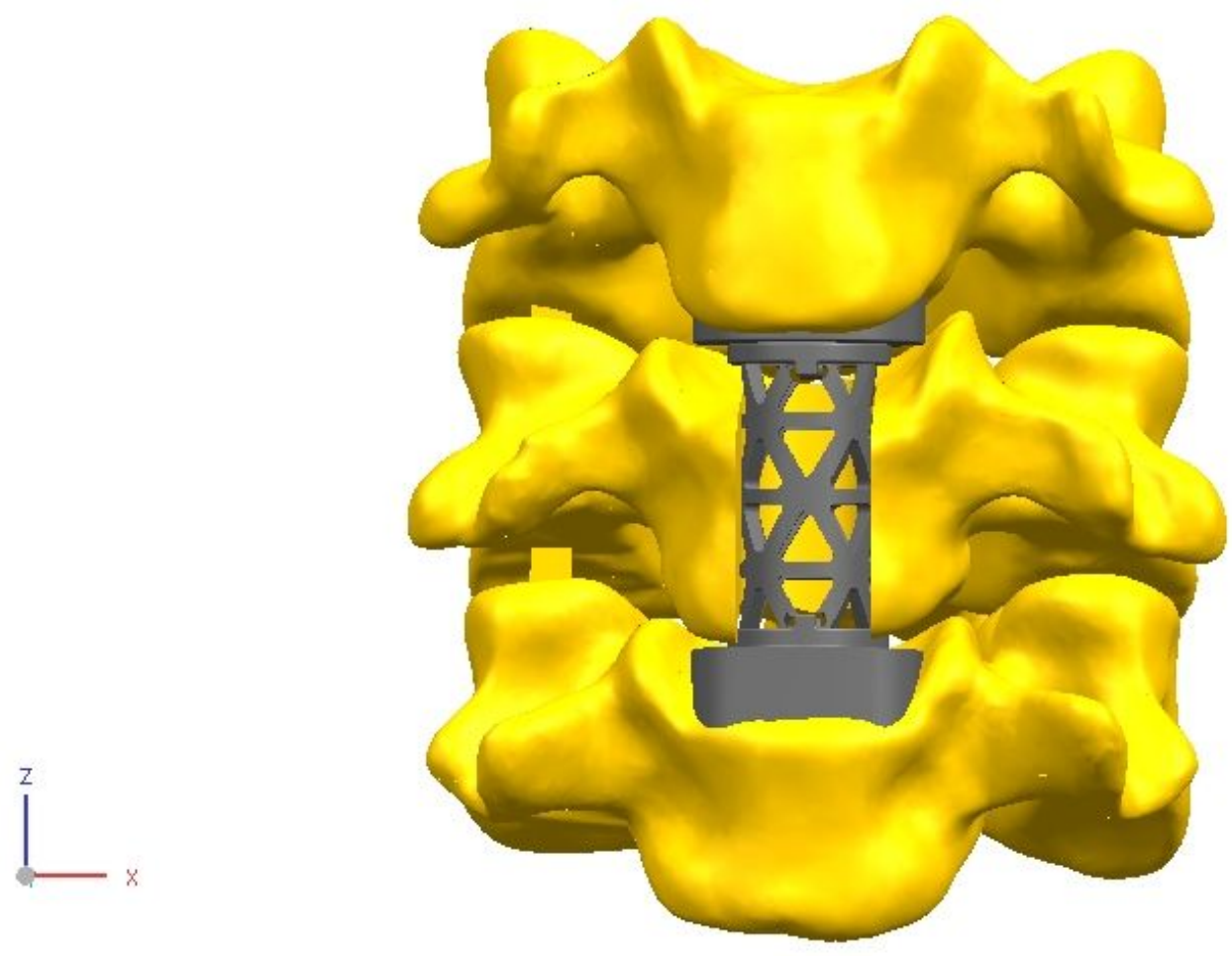

Figure 1

The novel anatomic titanium mesh cage for cervical spine.

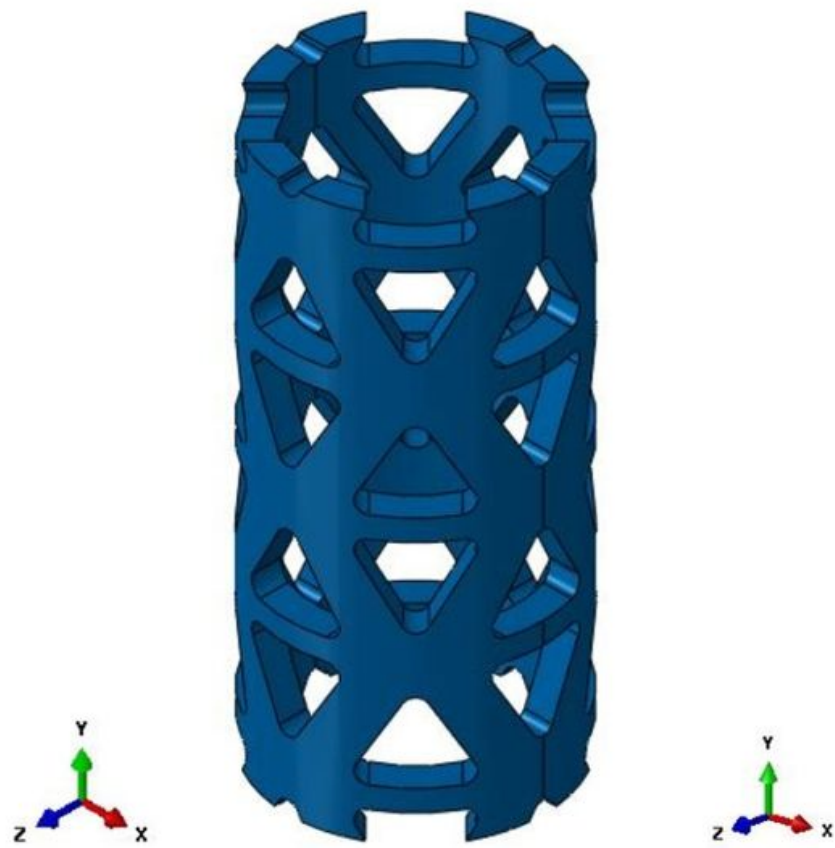

A

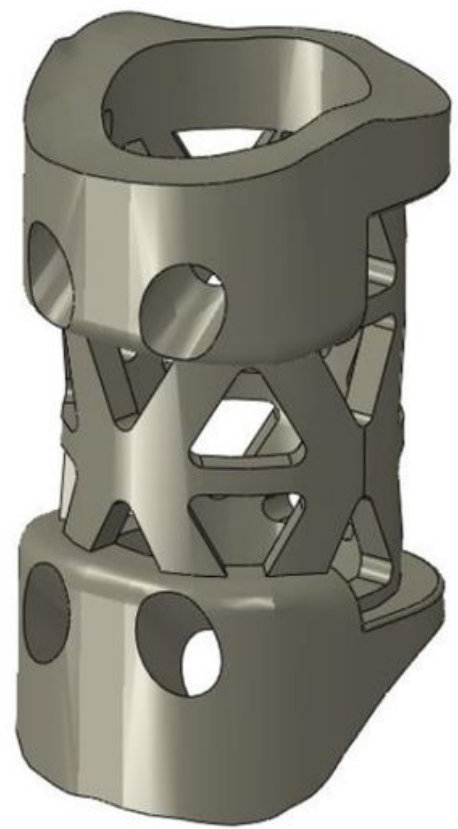

B

Figure 2 
Two TMC models. A TTMC; B NTMC.
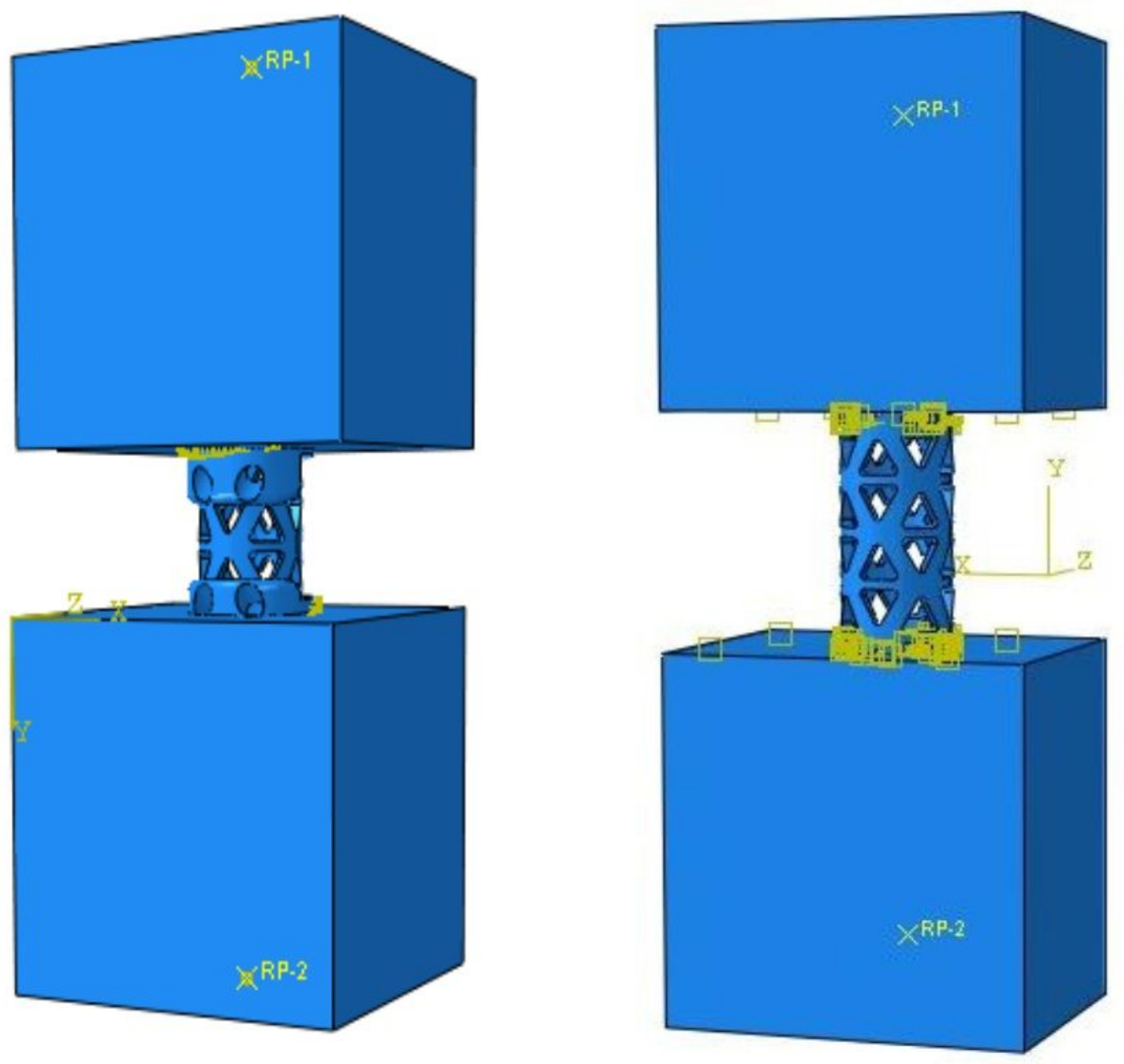

Figure 3

Schematic diagram of diagram of boundary conditions and load settings for NTMC and TTMC.

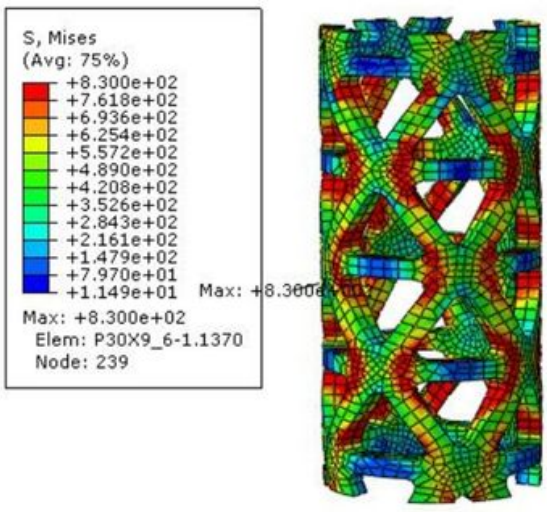

A

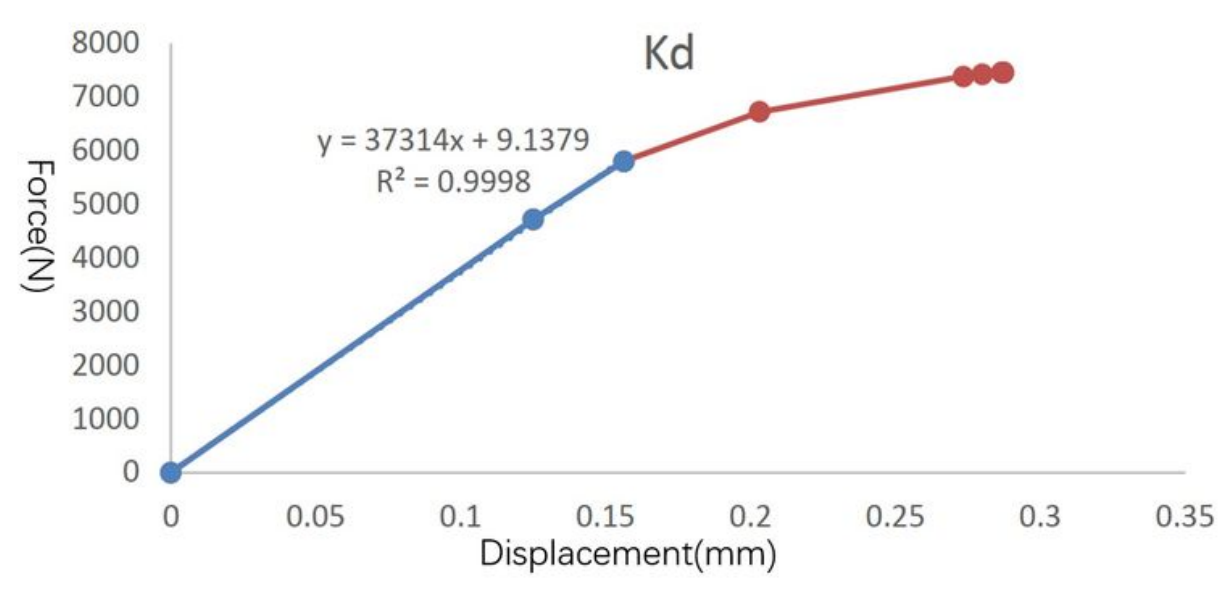

B

Figure 4 
The stress distribution and the force displacement curve of TTMC's Kd. A the stress distribution diagram; B the force displacement curve.
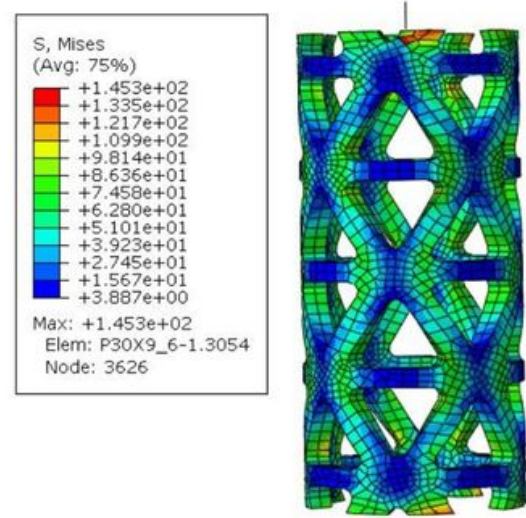

A

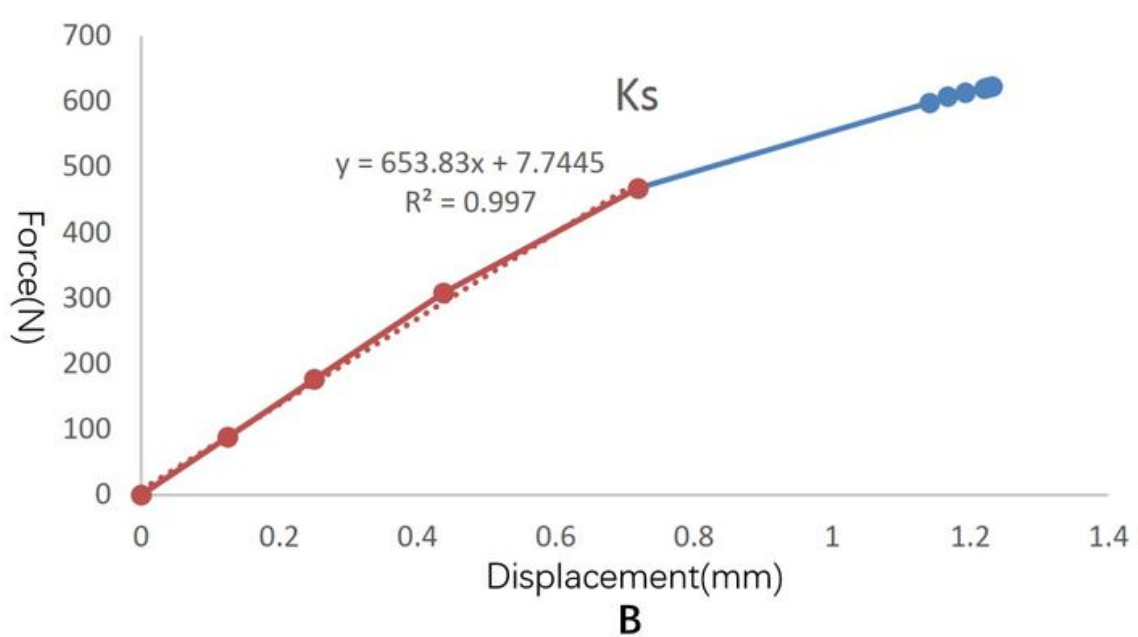

B

\section{Figure 5}

The stress distribution and the force displacement curve of TTMC's Ks. A the stress distribution diagram; $B$ the force displacement curve.
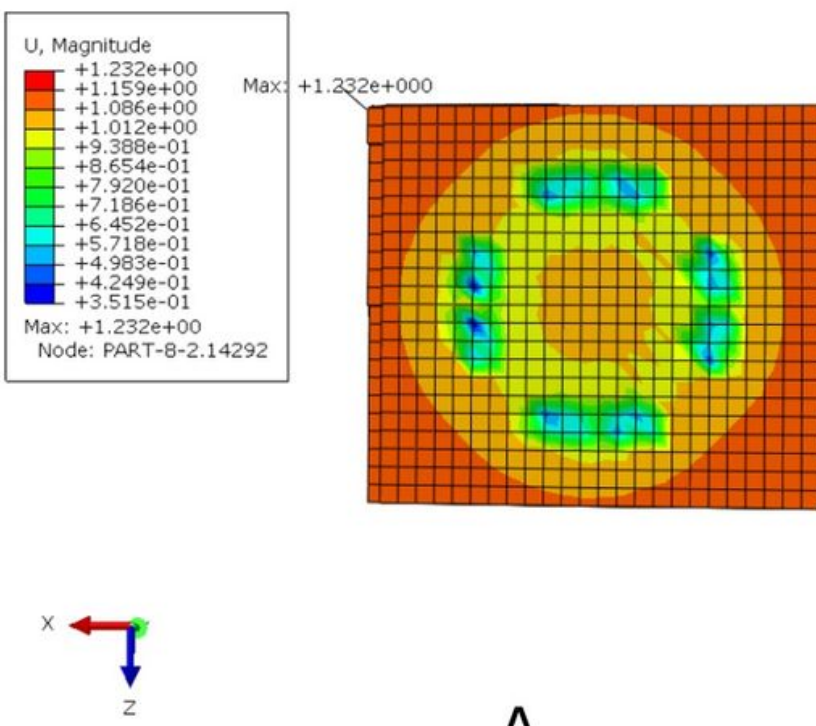
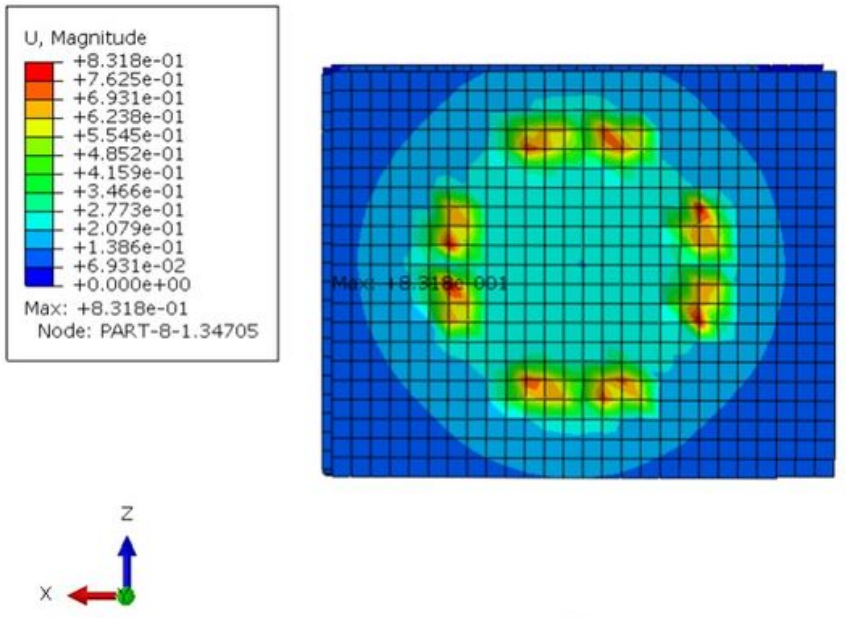

B

\section{Figure 6}

The subsidence and displacement nephogram of TTMC. A upper polyurethane foam blocks subsidence displacement nephogram; B lower polyurethane foam blocks subsidence displacement nephogram. 


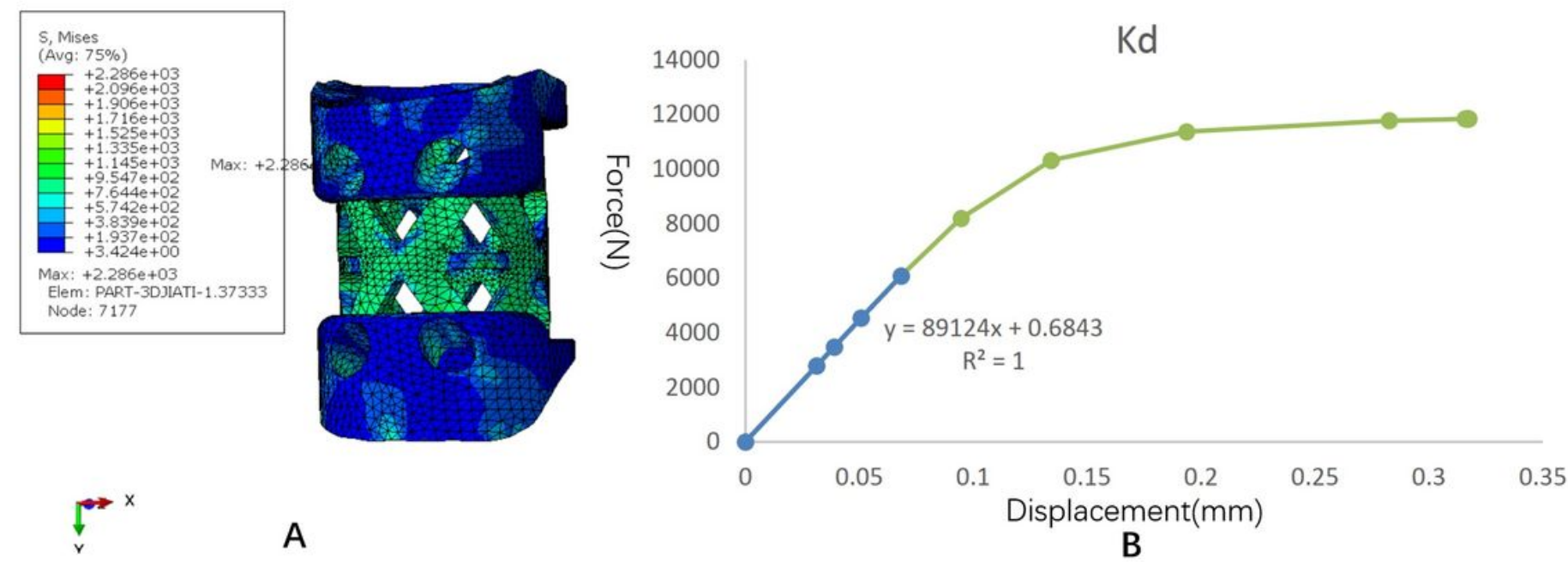

Figure 7

The stress distribution and the force displacement curve of NTMC's Kd. A the stress distribution diagram; B the force displacement curve.

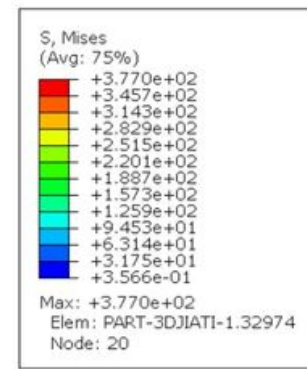

Node: 20

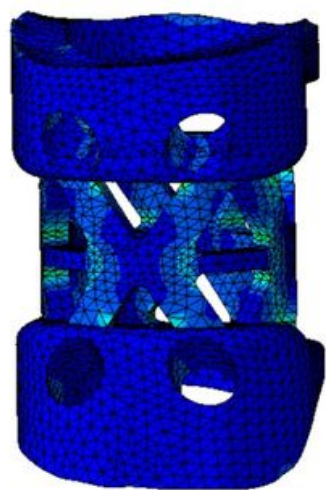

A

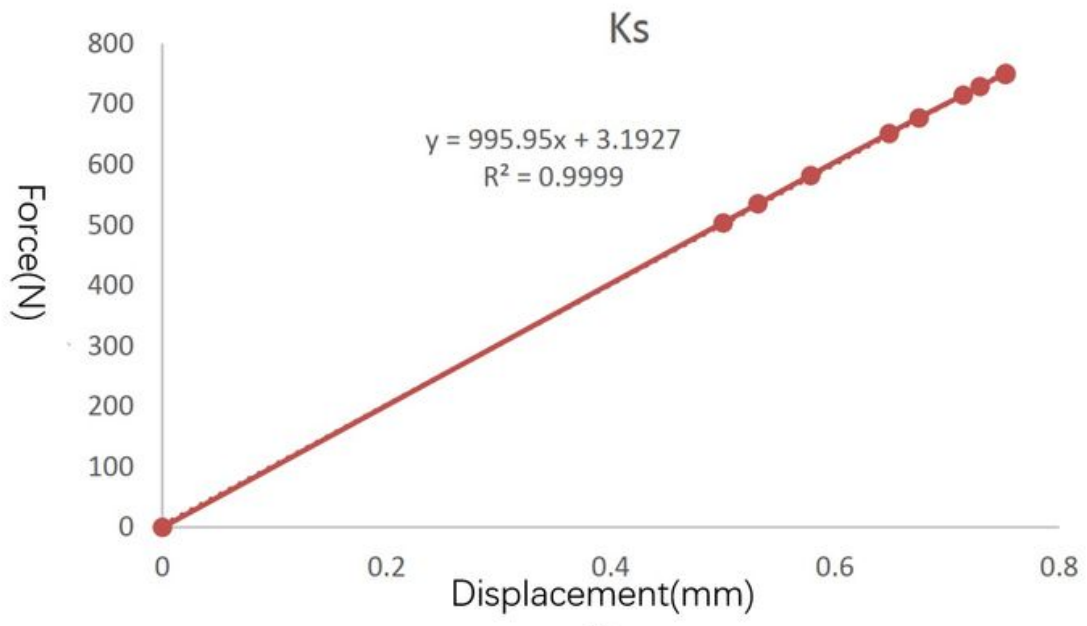

B

\section{Figure 8}

The stress distribution and the force displacement curve of NTMC's Ks. A the stress distribution diagram; $B$ the force displacement curve. 


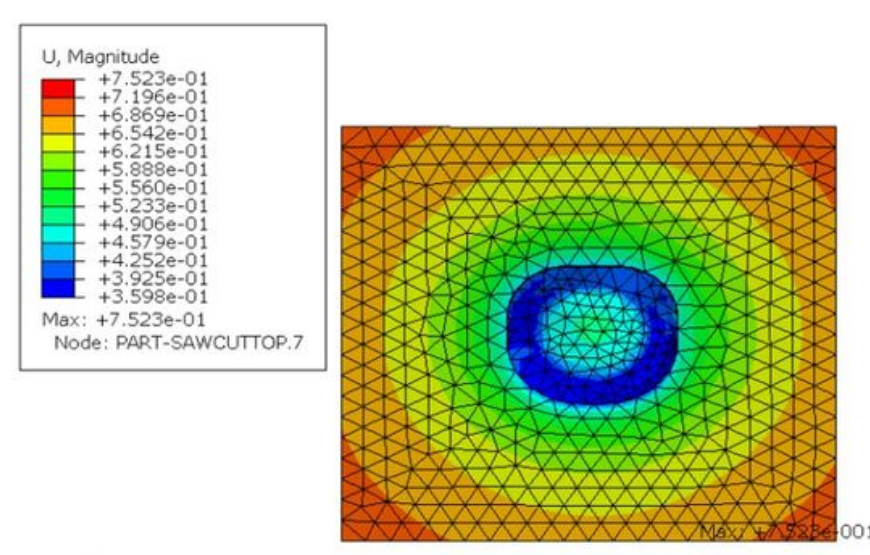

A

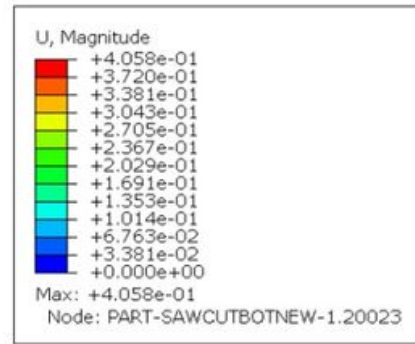

$x$

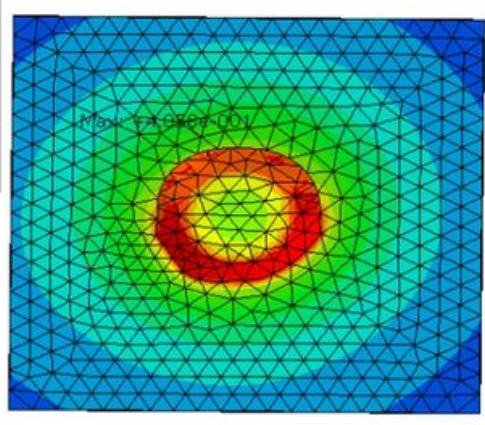

B

Figure 9

The subsidence and displacement nephogram of NTMC. A upper polyurethane foam blocks subsidence displacement nephogram; B lower polyurethane foam blocks subsidence displacement nephogram. 